\title{
Polímero de MMA para base de dentadura com a adição de subnitrato de bismuto
}

\author{
Addition of bismuth subnitrate in denture base MMA polymer
}

\author{
Stéfani Becker Rodrigues* \\ Vicente Castelo Branco Leitune** \\ Fabrício Mezzomo Collares ${ }^{* *}$ \\ Lina Naomi Hashizume ${ }^{* * *}$ \\ Susana Maria Werner Samuel ${ }^{* * * *}$
}

\section{Resumo}

Objetivo: o presente estudo tem por objetivo avaliar a influência da adição de diferentes concentrações de subnitrato de bismuto (SN) nas propriedades de materiais para base protética. Materias e método: foi adicionada $0 \%, 1 \%, 2 \%, 5 \%, 10 \%$ e $20 \%$ de $S \mathrm{~N}$ em peso em resina acrílica autopolimerizável da marca Clássico e submetidos aos ensaios de resistência à flexão, microdureza Knoop, radiopacidade, análise antimicrobiana, rugosidade e caracterização em micro-Raman. O teste estatístico escolhido foram o ANOVA de uma via e o teste de comparações múltiplas de Tukey. O nível de significância foi de 5\% para todos os testes. Resultados: os resultados de resistência à flexão atingiram valores acima do recomendado pela ISO 1567. Quanto maior a concentração da carga de SN maior o valor de microdureza Knoop, sendo estatisticamente significativo em $10 \%$ e $20 \%$ ( $p<0,001)$; os resultados da rugosidade mostraram valores abaixo do limiar, 0,2 $\mu \mathrm{m}$. A adição de subnitrato de bismuto não obteve um potencial antimicrobiano, mas apresentou uma maior radiopacidade, $(p<0,001)$. Conclusão: diante dos dados, conclui-se que a adição da carga de SN influencia nas propriedades mecânicas de resina acrílica significativamente, destacando-se que a radiopacidade desse material facilita a sua remoção quando ingerida ou aspirada.

Palavras-chave: Bismuto. Polimetil metacrilato. Radiopacidade.

\section{Introdução}

$\mathrm{Na}$ Odontologia, as resinas acrílicas são utilizadas por apresentarem propriedades e características essenciais para as funções clínicas. Diversos dispositivos protéticos são confeccionados com resinas acrílicas, tais como dentaduras, coroas provisórias e aparelhos ortodônticos ${ }^{1-3}$. Entretanto, as resinas acrílicas ainda manifestam algumas limitações como a radiolucidez e a porosidade que podem levar ao acúmulo de microrganismos ${ }^{4}$.

Em casos de acidentes traumáticos com dispositivos de resina acrílica, existem relatos de penetração de fragmentos em tecido mole do paciente ou de aspiração. Por não ser um material com características radiopacas, sua remoção pode tornar-se demorada devido à dificuldade de localização por radiografia. Dos pacientes que usam próteses dentárias removíveis $^{5}$, de $30 \%$ a $60 \%$ apresentam estomatites protéticas, condição inflamatória crônica associada com a mucosa oral afetada ${ }^{6,7}$. Assim, a adição de substâncias que possam melhorar a radiopacidade e a capacidade antimicrobiana das resinas acrílicas são desejadas.

O subnitrato de bismuto (SN) que, além de ser radiopaco, tem potencial antimicrobiano contra Helicobacter pylori ${ }^{8}$ pode ser uma alternativa de

\footnotetext{
Mestre em Odontologia - materiais Dentários, Departamento de Odontologia Conservadora, Porto Alegre, Rio Grande do Sul, Brasil. Doutor, professor adjunto da FO-UFRGS, Departamento de Odontologia Conservadora, Porto Alegre, Rio Grande do Sul, Brasil.

*** Doutora, professora adjunta da FO-UFRGS, Departamento de Odontologia Preventiva e Social, Porto Alegre, Rio Grande do Sul, Brasil.

**** Doutora, professora titular da FO-UFRGS, Departamento de Odontologia Conservadora, Porto Alegre, Rio Grande do Sul, Brasil.
} 
incremento dessas propriedades. Entretanto, esse material ainda não foi adicionado à resina acrílica para base de prótese total. Assim, o objetivo desse estudo é avaliar a influência da adição de diferentes concentrações de SN nas características de materiais para base de prótese, bem como seu potencial antimicrobiano.

\section{Materiais e método}

Este estudo foi realizado no Laboratório de Materiais Dentários (Lamad) e no Laboratório de Bioquímica e Microbiologia Bucal (LABIM) da Faculdade de Odontologia da UFRGS. Adicionou-se 0\%, 1\%, $2 \%, 5 \%, 10 \%$ e $20 \%$ de SN em peso em resina acrílica autopolimerizável da marca Clássico (Artigos Odontológicos Clássicos ${ }^{\circledR}$, São Paulo, SP, Brasil).

\section{Confecção dos corpos de prova}

O material foi confeccionado de acordo com as orientações do fabricante (2-pó:1-líquido, em massa), em que a proporção pó/líquido foi mensurada em uma balança analítica de precisão (AUW220D, Shimadzu, Tokyo, Japão). Após a manipulação, a resina acrílica autopolimerizável foi inserida em uma matriz metálica ou de polivinilsiloxano. Sobre a resina autopolimerizável foi colocada uma película de polietileno e sobre o conjunto, um peso de $300 \mathrm{~g}$.

\section{Cálculo da amostra}

O número amostral de cada ensaio foi calculado por meio do programa de análise estatística Sigma Stat. Para um nível de significância de $5 \%$ e um poder de estudo de $80 \%$ foi utilizado. Os parâmetros utilizados para o cálculo de cada metodologia foram: diferença mínima detectável, desvio-padrão estimado dos resíduos e número de grupos. Os valores foram baseados em estudos prévios realizados pelo laboratório. Nos ensaios que apresentam normatização da ISO, o número amostral seguirá a recomendação da especificação.

\section{Análise Microbiológica}

Três corpos de prova foram confeccionados para cada grupo (SN 0\%, SN 5\% e SN 20\%), totalizando 9 corpos de prova, com as dimensões de $8 \mathrm{~mm}$ de diâmetro e $2 \mathrm{~mm}$ de espessura para a análise microbiológica. Após, foram lixados com lixas d'água de granulação 600 e mantidos por $12 \mathrm{~h}$ a $37^{\circ} \mathrm{C}$ em água destilada para remoção do monômero residual ${ }^{9,10}$. Culturas puras de Candida albicans (ATCC 18804) foram crescidas em placas de Ágar Saboraud com cloranfenicol (Neogen Corporation, Michigan, EUA) a $25{ }^{\circ} \mathrm{C}$. Os corpos de prova foram esterilizados em plasma de hidrogênio e preparados por incubação em saliva clarificada, através de unidades filtrantes (0,2 $\mu \mathrm{m}$; Millipore Corporation, Billerica, EUA) por
30 min a $37^{\circ} \mathrm{C}$. Após 24 h, as colônias foram suspendidas em microtubos, contendo $2 \mathrm{~mL}$ de caldo Brain Heart Infusion (HiMedia Laboratories Pvt.Ltd, Mumbai, Índia). A suspensão celular foi ajustada através de um espectrofotômetro em 0,32 a $520 \mathrm{~nm}$ de absorbância ${ }^{10}$. Após os espécimes serem removidos da saliva, eles foram colocados em microtubos com a suspensão celular ajustada de $C$. albicans. Esses meios de cultura contendo os corpos de prova foram incubados a $37^{\circ} \mathrm{C}$ durante $72 \mathrm{~h}$ para a formação de biofilme. Após período de formação de biofilme, os corpos de prova (LAMAD) foram colocados em tubos com solução salina tamponada com fosfato (PBS) estéril. Os microrganismos aderidos aos corpos de prova foram removidos por sonicação a $7 \mathrm{~W}$ por 30s. A solução sonicada foi serialmente diluída e plaqueada em triplicata em meio de cultura Ágar Saboraud com cloranfenicol (Neogen Corporation, Michigan, EUA). As placas foram incubadas a $37^{\circ} \mathrm{C}$ por $72 \mathrm{~h}$ em aerobiose. Após esse período de incubação, as unidades formadoras de colônia foram contadas através de um microscópio estereoscópico (Olympus ${ }^{\circledR}$ CX21, Tokyo, Japão) e os resultados foram expressos em unidades formadoras de colônia (UFC) por área de C. albicans.

\section{Microdureza Knoop}

Um microdurômetro automático HMV-2 (Shimadzu $^{\circledR}$, Tokyo, Japão) foi utilizado para determinar a microdureza Knoop. Foram confeccionados cinco corpos de prova por grupo $(n=5)$, totalizando 30 corpos de prova, com dimensões de $10 \mathrm{~mm}( \pm 0,5$ $\mathrm{mm})$ de diâmetro por $1,0 \mathrm{~mm}( \pm 0,2 \mathrm{~mm})$ de espessura. Posteriormente, foram lixados com lixas d'água de granulação 400, 600 e 1000, foram ainda polidos com disco de feltro com pasta de óxido de alumínio. Os valores de microdureza foram obtidos através de três medidas realizadas na superfície de cada um dos corpos de prova, uma no centro e as demais distantes $100 \mu \mathrm{m}$ do centro.

$\mathrm{O}$ valor de microdureza Knoop (KHN) foi obtido por meio da medida da diagonal maior (d) de uma penetração losangular, deixada pelo penetrador de diamante de formato piramidal. O valor da diagonal foi medido em $\mu \mathrm{m}$, a partir de uma escala na ocular do microscópio. A carga aplicada (c) foi de $25 \mathrm{~g}$ durante 10s. O cálculo da microdureza Knoop foi realizado de acordo com a equação que segue (1):

$$
\mathrm{KHN}=\left[(14228 \mathrm{c}) /\left(\mathrm{d}^{2}\right)\right]
$$

em que: 14228 é o valor de uma constante; c é a carga aplicada em gramas; $d$ é a diagonal maior deixada pela penetração em $\mu \mathrm{m}$. O valor da microdureza Knoop foi expresso em número absoluto.

\section{Resistência à flexão}

Os corpos de prova para o ensaio de resistência à flexão, cinco de cada grupo $(n=5)$ com um total de 30 , foram produzidos de acordo com a especificação 
1567 da Internacional Organization of Standardization 11 com $64 \mathrm{~mm}$ de comprimento por $10 \mathrm{~mm}$ altura e por $3 \mathrm{~mm}$ de espessura, utilizando uma matriz de aço inoxidável. Foram lixados com lixas d'água número 400, 600 e 1000, polidas com disco de feltro e pasta de óxido de alumínio. Uma carga crescente a partir de zero, com velocidade constante de $5 \mathrm{~mm} / \mathrm{min}$ ( $\pm 1 \mathrm{~mm} / \mathrm{min})$, foi aplicada no centro de cada corpo-de-prova, que se encontrava apoiado nas duas extremidades. A carga foi aplicada até a ruptura dos corpos de prova. A resistência à flexão $(\sigma)$ foi calculada, em megapascal (MPa), de acordo com a equação a seguir (2):

$$
\sigma=3 \mathrm{FL} / 2 \mathrm{BH}^{2}
$$

em que $\mathrm{F}$ é a força máxima, em Newtons; $\mathrm{L}$ é a distância, em milímetros, entre os suportes; B é a largura, em milímetros, da amostra medida imediatamente antes do teste; $\mathrm{H}$ é a altura em milímetros.

\section{Rugosidade}

A rugosidade foi aferida em três corpos de prova por grupo $(n=3)$, com total de 18 , com dimensões de $8 \mathrm{~mm}$ de diâmetro por $2 \mathrm{~mm}$ de espessura. Após, foram lixados com lixas d'água de granulação 400 , 600 e 1000 e polidos com disco de feltro com pasta de óxido de alumínio. A análise da rugosidade dos corpos de prova foi realizada através de um rugosímetro digital SJ-201 (Mitutoyo ${ }^{\circledR}$, Kawasaki-shi, Kanagawa, Japão). Esse aparelho contém um sensor, que ao percorrer a superfície do material, atribui valores, que definem picos e vales presentes nessa superfície. O valor atribuído à área de picos e de vales é dividido pela distância percorrida pelo sensor em linha reta, fornecendo o parâmetro de rugosidade $\mathrm{Ra}$ em $\mu \mathrm{m}$. $\mathrm{O}$ aparelho forneceu a média de três percursos de $0,25 \mu \mathrm{m}$.

\section{Radiopacidade}

A fim de avaliar a radiopacidade, foram confeccionados cinco corpos de prova por grupo $(n=5)$, no total de 30 corpos de prova com $10 \mathrm{~mm}( \pm 0,5 \mathrm{~mm})$ de diâmetro por $1,0 \mathrm{~mm}( \pm 0,2 \mathrm{~mm})$ de espessura, utilizando uma matriz de polivinilsiloxano. Os exames radiográficos de todos os grupos foram realizados com o sistema digital com placas de fósforo VistaScan (Dürr Dental GmbH \& CO.KG, Bietigheim-Bissingen, Alemanha) em regime de $70 \mathrm{kVp}$, $8 \mathrm{~mA}, 0,6$ segundos de exposição e distância focal de $400 \mathrm{~mm}$. Sobre a placa foi posicionado um corpo-de-prova de cada grupo, totalizando seis amostras em cada placa. Uma escala de alumínio foi posicionada juntamente com os corpos de prova em todas as tomadas radiográficas. A escala de alumínio utilizada é composta por no mínimo $98 \%$ de alumínio e apresenta espessura que varia de $0,5 \mathrm{~mm}$ até 9,0 $\mathrm{mm}$, com incrementos de $0,5 \mathrm{~mm}$. As imagens resultantes foram transferidas para o computador, utilizando o scaner do sistema VistaScan, armazenadas em disco no formato TIFF e analisadas no programa Fotoshop CS (Adobe Systems Incorporated, San Jose, Califórnia, EUA). Os valores médios e os desvios-padrão dos níveis de cinza (em densidade de pixels) dos corpos de prova e da escala de alumínio foram obtidos em uma área padronizada por corpo-de-prova, previamente estabelecida de $8 \mathrm{~mm}^{2}$. O ensaio foi repetido em cinco corpos de prova de cada formulação experimental e a radiopacidade equivalente foi expressa em milímetros de alumínio ${ }^{12}$.

\section{Caracterização em micro-Raman}

Uma espectrofotometria Micro-Raman foi realizada, utilizando um microscópio SEN- TERRA Raman (Bruker Optics, Ettlingen, Alemanha). A amostra foi analisada usando um parâmetro de micro-Raman: laser de diodo a $100 \mathrm{~mW}$ com comprimento de onda de $785 \mathrm{~nm}$ e resolução de espectro de $\sim 3.5 \mathrm{~cm}-1$. Um mapeamento unidimensional foi feito por meio de uma linha de $70 \mathrm{~mm}$ sobre a área central do corpo-de-prova com $1 \mathrm{~mm}$ de intervalo, usando um estágio computadorizado XYZ. O tempo de acumulação por espectro foi de $5 \mathrm{~s}$ com duas co-adições. Realizou-se um mapeamento com uma amostra de resina acrílica com $20 \%$ de subnitrato de bismuto em locais aleatórios ${ }^{13}$.

\section{Análise dos resultados}

A normalidade dos valores obtidos foi testada através do teste Kolmogorov-Smirnov. O teste estatístico escolhido foi o ANOVA de uma via e, havendo diferença, foi aplicado o teste de comparações múltiplas de Tukey. O nível de significância foi de 5\% para todos os testes.

\section{Resulltados}

Os resultados da análise microbiológica estão representados na Tabela 1. Não houve diferença estatisticamente significativa entre os grupos $0 \%, \mathrm{SN}$ $5 \%$ e SN $20 \%(\mathrm{p}=0,087)$.

Tabela 1 - Valores da análise microbiológica expressa em unidades formadoras de colônia

\begin{tabular}{l|c}
\hline \multicolumn{1}{c|}{ Concentração } & UFC $/ \mathrm{mL}$ \\
\hline $0 \%$ & $3,6( \pm 0,1)^{\mathrm{A}}$ \\
\hline $5 \%$ & $2,9( \pm 0,5)^{\mathrm{A}}$ \\
\hline $20 \%$ & $2,9( \pm 0,2)^{\mathrm{A}}$ \\
\hline
\end{tabular}

Letras maiúsculas diferentes indicam diferença estatística $(p<0,05)$ na mesma coluna.

Para o ensaio de microdureza Knoop (Figura 1), os valores variaram entre 15,9 no grupo SN $0 \%$ e 20,7 no grupo SN $20 \%$, o poder do estudo foi de 99,2 $\%$. Não houve diferença significativa entre os grupos $0 \%, 1 \%, 2 \%, 5 \%$, e $10 \%$, entretanto, houve com o grupo SN $20 \%,(\mathrm{p}<0,001)$. 


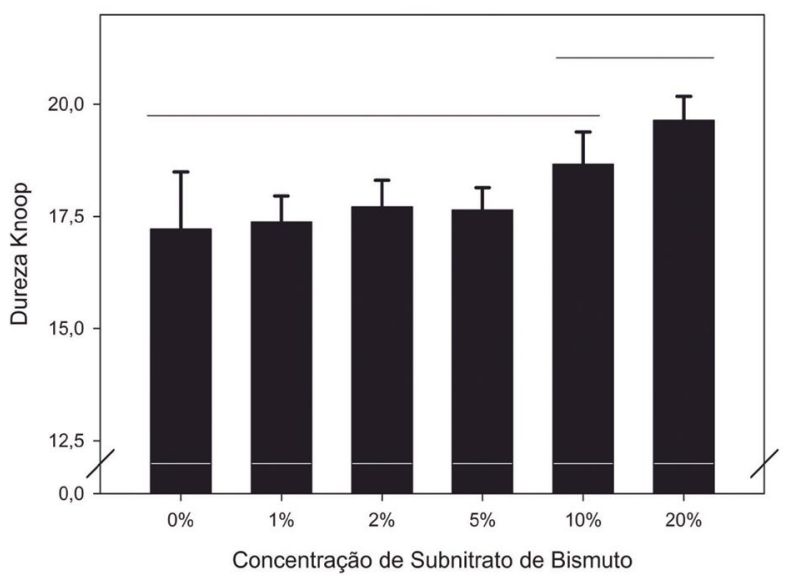

Figura 1 - Resultados do ensaio de microdureza Knoop

Os valores de rugosidade de todos os grupos ficaram abaixo de 0,2 $\mu \mathrm{m}$ (Figura 2), com $\mathrm{p}<0,001 \mathrm{e}$ poder do estudo de $99,2 \%$.

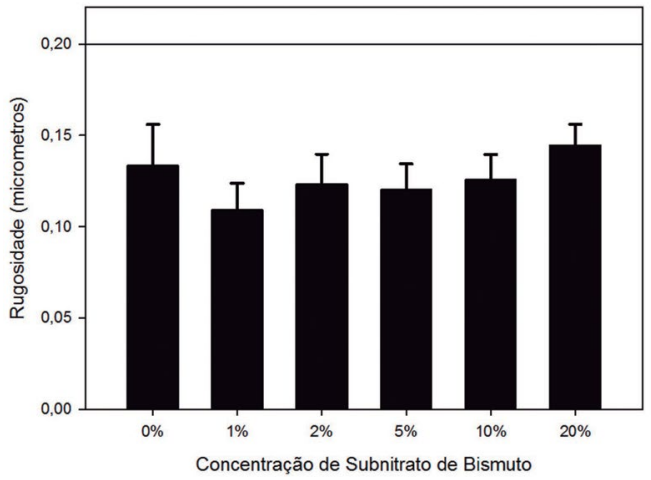

Figura 2 - Resultados da rugosidade em micrometros

Para o teste de radiopacidade, Figura 3, quanto maior a concentração de subnitrato de bismuto, maior o valor encontrado $(p<0,001)$. Entretanto, não houve diferença entre os grupos de SN $0,1 \%$ e $2 \%$, havendo somente entre o grupo de controle (0\%) e os grupos com adição de $5 \%, 10 \%$ e $20 \%$ de subnitrato de bismuto.

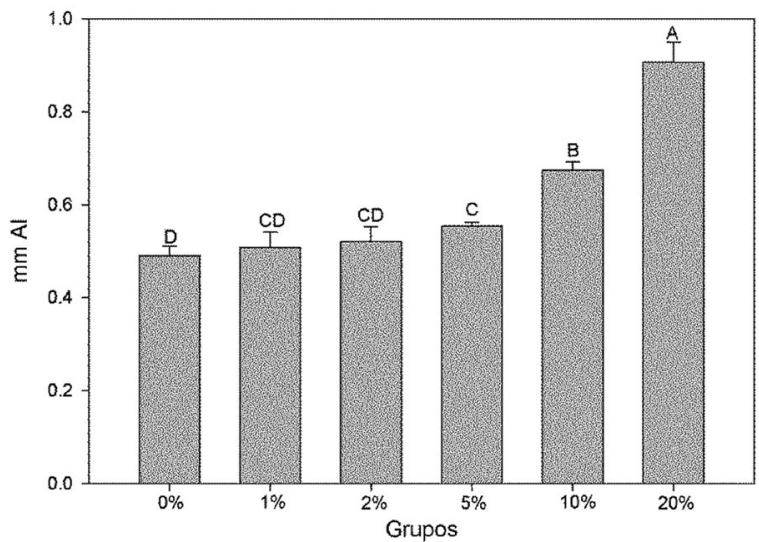

Figura 3 - Avaliação da radiopacidade da resina acrílica expressa em $\mathrm{mm}$ de alumínio

Apesar de o grupo com adição de $20 \%$ de subnitrato de bismuto apresentar valor médio de resistência à flexão inferior ao preconizado pela ISO
1567, nenhum grupo com adição de carga apresentou diferença estatisticamente significativa em relação ao grupo de controle (0\%), de acordo com o apresentado na Figura 4. O poder do estudo foi de $90 \%$, com $\mathrm{p}=0,003$.

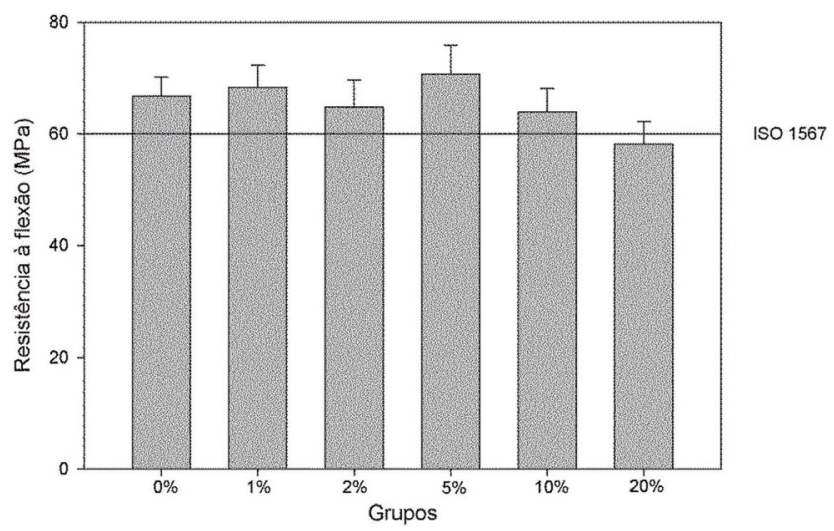

Figura 4 - Resistência à flexão da resina acrílica

A Figura 5 apresenta o mapeamento, por meio da espectroscopia micro-Raman, da superfície da resina acrílica com subnitrato de bismuto. A distribuição homogênea das partículas pode ser percebida em toda a superfície da área analisada.

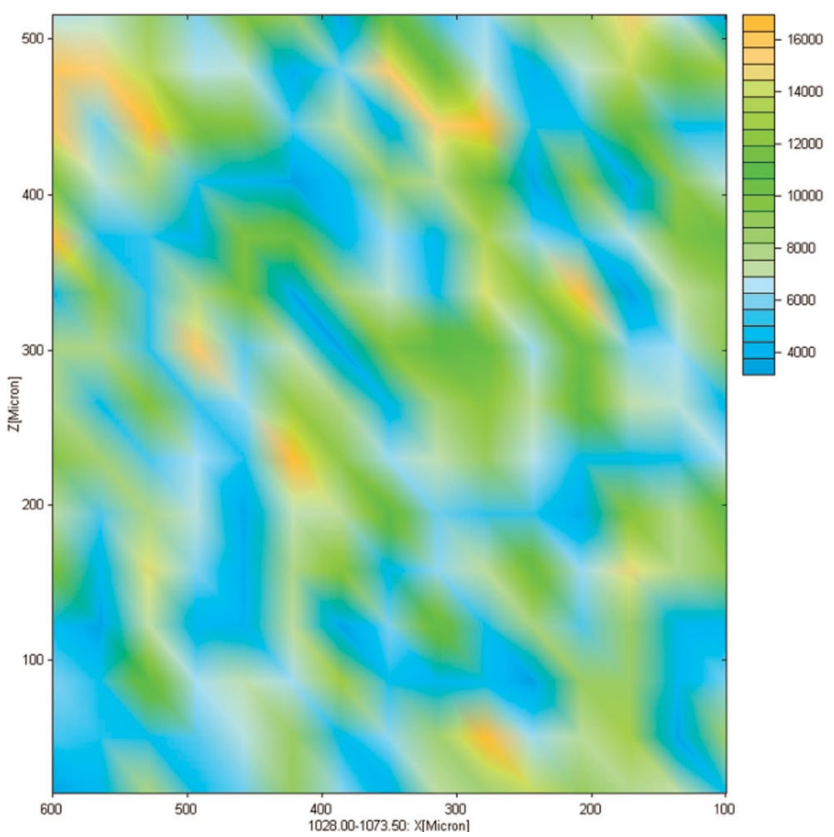

Figura 5 - Mapeamento por micro Raman da superfície da resina acrílica com $20 \%$ de Subnitrato de Bismuto

\section{Discussão}

As melhorias das propriedades mecânicas em materiais para a confecção de próteses removíveis induzem a busca de novos materiais. Devido ao fato de o Subnitrato de Bismuto por (SN) constituir uma carga com alto número atômico e apresentar propriedade antimicrobiana, revela-se uma alternativa 
para inclusão em materiais odontológicos. A adição de $\mathrm{SN}$, neste trabalho, aumentou a dureza e a radiopacidade da resina acrílica sem influenciar negativamente na rugosidade superficial e na resistência à flexão. A distribuição das partículas se apresentou homogênea em toda a superfície da resina acrílica. Entretanto, independente da concentração, a resina com adição de carga não apresentou efeito antimicrobiano sobre a C. albicans.

A dispersão da incorporação de diferentes concentrações de SN na resina acrílica ocorreu de maneira homogênea. Por meio da espectroscopia Raman (Figura 5), observa-se essa mistura homogênea entre carga, concentração de SN $20 \%$ e resina acrílica. A adição de SN não modificou a incorporação do pó com o líquido durante a sua manipulação, como demonstrado por Rawls et al. ${ }^{14}$ (1990), quando adicionaram haletos de bismuto na matriz resinosa e por Lang et al. ${ }^{15}$ (2000), quando incorporaram trifenilbismuto também em resina acrílica, assim como conferiram radiopacidade à resina acrílica autopolimerizável. No presente estudo, as concentrações de $1 \%$ e $2 \%$ não apresentaram diferença com o grupo de SN 0\%, entretanto, acima de $5 \%$ obtiveram valores estatisticamente significativos do grupo com $0 \%$ de SN, tornando assim a resina acrílica autopolimerizável radiopaca.

Apesar de o grupo com adição de $20 \%$ de SN apresentar valor médio de resistência à flexão inferior ao preconizado pela ISO 1567, os valores médios de todos os grupos não apresentaram diferença estatisticamente significativa com o grupo de controle. A redução da resistência à flexão do grupo com $20 \%$ pode ter ocorrido pela alta concentração de carga no corpo-de-prova, ocorrendo alguns pontos de aglutinação, os quais agiram como áreas de concentração de tensão, facilitando a fratura. Os valores de microdureza Knoop entre os grupos de 0\%, 1\%, 2\%, 5\% e 10\% de SN não apresentaram diferença estatisticamente significativa, mostrando diferença apenas com o grupo que continha $20 \%$ de SN. Embora, a adição de cargas inorgânicas possa levar a um aumento na microdureza Knoop, no presente estudo, apenas o grupo com $20 \%$ de SN apresentou valores estatisticamente superiores ao grupo sem adição. A ausência de diferença entre os grupos com até $10 \%$ de carga pode ser explicada pela presença de cargas dispersas na matriz orgânica e quando o corpo-de-prova foi submetido à indentação, a matriz orgânica subjacente à carga cedeu, reduzindo os valores de dureza ${ }^{16}$.

Superfícies bem polidas são de extrema importância para o conforto do paciente e também para a longevidade da prótese, garantindo a estética, a higiene bucal e a baixa retenção de biofilme ${ }^{17}$. Assim, a adição de SN não modificou a rugosidade superficial dos corpos de prova, mesmo nas concentrações mais altas. Os resultados encontrados foram valores abaixo do limiar de rugosidade, $0,2 \mu^{18}$, tornando essa superfície mais difícil de ser colonizada por bactérias ${ }^{19}$.
Para que o limiar de rugosidade não interferisse na adesão microbiana, diferentes corpos de prova foram confeccionados para o ensaio antimicrobiano para que ocorresse a formação de biofilme durante 0 ensaio microbiológico, uma vez que os corpos de prova para a rugosidade estavam com valores de rugosidade abaixo do limiar. Sabe-se que a imersão do substrato em saliva provoca uma diminuição na rugosidade da superfície e na energia de superfície livre, diminuindo assim a quantidade de espécies de C. albicans nas amostras, o que se confirmou neste estudo.

Entretanto, mesmo com essa diminuição, não foi constatado que a resina acrílica, quando incorporada ao Subnitrato de Bismuto, seja antimicrobiana para a C. albicans. Provavelmente, a carga incorporada ficou aprisionada no interior da rede polimérica e não na superfície da resina acrílica, assim, como também, não houve lixiviação do material para que ocorresse um efeito antimicrobiano.

Apesar do SN não ter apresentado, neste estudo, potencial antimicrobiano, pode ser utilizado para melhorar a microdureza Knoop, resistência à flexão, radiopacidade, sem interferir na rugosidade da resina acrílica autopolimerizável. Assim, a utilização de uma resina acrílica radiopatizante teria como característica a sua fácil remoção, quando ingerida ou aspirada acidentalmente por pacientes que utilizam próteses removíveis.

\section{Conclusão}

A adição de SN em resinas acrílicas autopolimerizáveis influencia significativamente nas propriedades mecânicas, considerando que a radiopacidade desse material facilita a sua remoção em casos de tais resinas serem ingeridas ou aspiradas acidentalmente.

\section{Agradecimentos}

Os autores agradecem à Capes (Coordenação de Aperfeiçoamento de Pessoal de Nível Superior) pela bolsa de estudos.

\section{Abstract}

Objective: The aim of this study was evaluate the effect of adding different concentrations of bismuth subnitrate $(S N)$ on the properties of denture base materials. Materials and method: Was added $0 \%, 1 \%, 2 \%, 5 \%$, $10 \%$ and $20 \%$ weight of SN in acrylic resin (Classico) and subjected to the tests of flexural strength, Knoop hardness, radiopacity, antibacterial analysis, roughness and characterization of micro-Raman spectroscopy. The statistical test chosen was one-way ANOVA and Tukey. The significance level was 5\% for all tests. Results: The results of flexural strength values were above the recommended by ISO 1567, the higher the concentration load greater the Knoop hardness value, was statistically 
significant at 10 and 20\%, p<0.001; results showed roughness values below threshold roughness $0.2 \mu \mathrm{m}$. The addition of bismuth subnitrate did not receive an antimicrobial potential, but showed greater radiopacity, $p<0.001$. Conclusion: Thus, the addition of this load significantly influences the mechanical properties and radiopacity of this material which facilitates their removal when ingested or aspirated.

Keywords: Bismuth. Polymethyl methacrylate. Radiopacity.

\section{Referências}

1. León BLT, Del Bel Cury AA, Rodrigues Garcia RCM. Loss of residual monomer from resilient lining materials processed by different methods. Rev Odonto Ciência 2008; 23:215-9.

2. Bayraktar G, Guvener B, Bural C, Uresin Y. Influence of polymerization method, curing process, and length of time of storage in water on the residual methyl methacrylate content in dental acrylic resins. J Biomed Mater Res B: Appl Biomater 2006; 76:340-5.

3. Urban VM, Machado AL, Vergani CE, Giampaolo ET, Pavarina AC, Almeida FG, et al. Effect of water-bath post-polymerization on the mechanical properties, degree of conversion, and leaching of residual compounds of hard chairside reline resins Dent Mater 2009; 25:662-71.

4. Bettencourt AF, Neves CB, de Almeida MS, Pinheiro LM, Oliveira SA, Lopes LP, et al. Biodegradation of acrylic based resins: A review. Dent Mater 2010; 26(5):e171-80.

5. Radford DR, Sweet SP, Challacombe SJ, Walter JD. Adherence of Candida albicans to denture-base materials with different surface finishes. J Dent 1998; 26(7):577-83.

6. Nair RG, Samaranayake LP. The effect of oral commensal bacteria on candidal adhesion to denture acrylic surfaces. An in vitro study. APMIS,1996; 104(5):339-49.

7. Radford DR, Challacombe SJ, Walter JD. Denture plaque and adherence of Candida albicans to denture-base materials in vivo and in vitro. Crit Rev Oral Biol Med 1999; 10(1):99-116

8. Carvalho AF, Fiorelli LA, Jorge VN, Da Silva CM, De Nucci G, Ferraz JG, et al. Addition of bismuth subnitrate to omeprazole plus amoxycillin improves eradication of Helicobacter pylori. Aliment Pharmacol Ther 1998; 12(6):557-61.

9. Del Bel Cury AA, Rached RN, Ganzarolli SM. Microwave-cured acrylic resins and silicone-gypsum moulding technique. J Oral Rehabil 2001; 28(5):433-8.

10. Moura JS, da Silva WJ, Pereira T, Del Bel Cury AA, Rodrigues Garcia RC. Influence of acrylic resin polymerization methods and saliva on the adherence of four Candida species. J Prosthet Dent. 2006; 96(3):205-11.

11. International Standards Organization. ISO 1567: Dentistry-denture base polymers, Geneve;1999.

12. Collares FM, Ogliari FA, Lima GS, Fontanella VR, Piva E, Samuel SM. Ytterbium trifluoride as a radiopaque agent for dental cements. Int Endod J 2010; 43(9):792-7.

13. Leitune VC, Collares FM, Takimi A, de Lima GB, Petzhold CL, Bergmann CP, et al. Niobium pentoxide as a novel filler for dental adhesive resin. J Dent. 2013;41(2):106-13.

14. Rawls HR, Starr J, Kasten FH, Murray M, Smid J, Cabasso I. Radiopaque acrylic resins containing miscible heavy-metal compounds. Dent Mater 1990; 6:250-5.
15. Lang LA, Mattie PA, Rawis HR. The effect of triphenylbismuth on the radiopacity and performance properties of compression- and injection-molded denture resins. prosthodontics 2000; 9(1):23-9.

16. Krumova M, Klingshirn C, Haupert F, Friedrich K. Microhardness studies on functionally graded polymer composites. Composites Science and Technology 2001; 61(4):557-63.

17. Pereira-cenci T, Del Bel Cury AA, Crielaard W, Ten Cate JM. Development of candida-associated denture stomatitis: new insights. J Appl Oral Sci 2008; 16(2):86-94.

18. Bollen CM, Lambrechts P, Quirynen M. Comparison of surface roughness of oral hard materials to the threshold surface roughness for bacterial plaque retention: a review of the literature. Dent Mater 1997;13(4):258-69.

19. Quirynen M, Marechal M, Busscher HJ, Weerkamp AH, Darius $\mathrm{PL}$, van Steenberghe D. The influence of surface free energy and surface roughness on early plaque formation. An in vivo study in man. J Clin Periodontol 1990; 17(3):138-44.

\section{Endereço para correspondência:}

Fabrício Mezzomo Collares

Ramiro Barcelos, 2492 - Laboratório de Materiais Dentários 90035-003 Porto Alegre-RS

Fone: (51)-3308-5198,

Fax: (51)-3308-5197

E-mail: fabricio.collares@ufrgs.br

Recebido: 14/11/2013. Aceito: 06/08/2014 